\title{
Renal Parenchymal Disease Mimicking Preeclampsia
}

\author{
Sashikala Paul ${ }^{1}$ Indrani Garre ${ }^{2}$ Kumar Achukatla² \\ ${ }^{1}$ Department of Gynecology and Obstetrics, Vijaya Marie Hospital, \\ Veernagar, Saifabad, Hyderabad, Telangana, India \\ 2Department of Cardiology, Nizam's Institute of Medical Sciences \\ (NIMS), Punjagutta, Hyderabad, Telangana, India
}

\begin{abstract}
Address for correspondence Indrani Garre, PhD Student, Department of Cardiology, Nizam's Institute Of Medical Sciences (NIMS), Punjagutta 500018, Hyderabad, Telangana, India (e-mail: Indrani.garre@gmail.com).
\end{abstract}

Indian J Cardiovasc Dis Women-WINCARS 2018;3:209-212

\section{Introduction}

Preeclampsia is defined as detection of systematic detection of hypertension (HTN) (blood pressure [BP] > 140/90 mm $\mathrm{Hg}$ ) on or after the 20th week of pregnancy with evidence of damage to the other organ systems. This pregnancy disorder is mainly associated with primigravida. There are genetic studies being performed to predict which women are likely to develop preeclampsia. ${ }^{1,2}$ We have to always find out the cause of HTN at whatever stage of the patient we see, and then we label them as essential HTN. Here, we report a case mimicking preeclampsia but turning out to be a chronic HTN-complicated pregnancy.

\section{Case Description}

A 28-year-old woman came to cardiology OP for evaluation of uncontrolled systemic hypertension (HTN). HTN was detected in the fifth month of pregnancy. She was a primi with maximum recorded BP 210/120 mm Hg. She was evaluated further at that time by a gynecologist. She had proteinuria with a range of $1.2 \mathrm{~g} /$ day, normal platelet count, and normal liver enzymes during pregnancy. The patient continued to take nifedipine (long acting) $20 \mathrm{mg}$ twice a day for control of HTN. At 36 weeks of pregnancy after confirmation of maturity of the fetus, a cesarean section was done by the attending obstetrician. The surgical procedure was uneventful.

Birth weight of the child was $2.5 \mathrm{~kg}$ with normal APGAR score. However, the mother's BP continued to rise after surgery, which increased up to 220/140 mm Hg despite drug therapy. Then the patient was referred to a cardiologist for further management, 15 days after delivery.

There was no history of convulsions, altered sensorium, and bleeding diathesis during pregnancy. The patient's BP was not tested before pregnancy. High BP (140/90 mm $\mathrm{Hg}$ ) was detected for the first time in the fifth month of pregnancy during antenatal checkups, which gradually increased to 220/120 mm Hg with pedal edema and dyspnea of class II. The patient was hospitalized for control of HTN and evaluated for preeclampsia, but there was no mention about ultrasound of the abdomen. Subsequently, with nifedipine, HTN crisis was controlled. The patient continued to have mild HTN throughout pregnancy $(130 / 90 \mathrm{~mm} \mathrm{Hg})$ with drugs. Cesarean section was done uneventfully without any HTN surge during or immediately after delivery.

There was an increase in systolic and diastolic BP without any signs and symptoms of pulmonary edema 15 days after delivery. We further evaluated the secondary causes of HTN. The patient was mildly anemic on examination. There was no cyanosis and respiratory distress. BP recording in all four limbs were as follows: right upper limb, 180/120 mm Hg; left lower limb, 190/130; right lower limb, 180/110; and left lower limb, 190/110.

Clinically, there was no evidence of coarctation of the aorta, renal bruit, or signs suggestive of systemic manifestations of collagen vascular disease. There was mild pitting pedal edema with a normal jugular venous pulse. Laboratory investigations showed hypoproteinemia, proteinuria, lower level of normal 
platelet count, mild anemia abnormal liver function tests, and increased erythrocyte sedimentation rate (ESR) ( - Table $\mathbf{1})$.

Electrocardiogram showed left ventricular hypertrophy (LVH) without strain ( - Fig. 1). Echo showed concentric LVH, confirming the long-standing HTN.

Table 1 Biochemical parameters of the patient

\begin{tabular}{|c|c|c|}
\hline Investigations & Result & Normal range \\
\hline Hemoglobin (g/dL) & 11.7 & $13-17$ \\
\hline PCV (vol\%) & 35 & - \\
\hline TLC (cells/mm³) & 13,600 & $4,000-11,000$ \\
\hline Platelet count (lakh/mm³) & 1.4 & $1.4-4.8$ \\
\hline Plasma glucose (mg/dL) & 136.2 & $70-100$ \\
\hline Serum sodium (mmol/L) & 138 & $135-145$ \\
\hline Serum potassium (mmol/L) & 3.7 & $3.5-5$ \\
\hline Serum chloride (mmol/L) & 102 & $95-106$ \\
\hline Serum creatinine $(\mathrm{mg} / \mathrm{dL})$ & 0.71 & - \\
\hline Serum uric acid (mg/dL) & 4.4 & $2.6-6$ \\
\hline Serum AST (U/L) & 55.4 & $<35$ \\
\hline Serum ALT (U/L) & 45.3 & $<40$ \\
\hline Serum ALP (U/L) & 210 & $<130$ \\
\hline Serum bilirubin (total) $(\mathrm{mg} / \mathrm{dL})$ & 0.26 & $<1.1$ \\
\hline $\begin{array}{l}\text { Serum bilirubin (conjugated) } \\
\text { (mg/dL) }\end{array}$ & 0.08 & $<0.2$ \\
\hline Serum total protein $(\mathrm{g} / \mathrm{dL})$ & 5.29 & $6-8$ \\
\hline Serum albumin $(\mathrm{g} / \mathrm{dL})$ & 2.4 & $3.5-5.2$ \\
\hline Serum LDH $(U / L)$ & 484 & $125-220$ \\
\hline
\end{tabular}

Abbreviations: ALP, alkaline phosphatase; ALT, alanine aminotransferase; AST, aspartate aminotransferase; LDH, lactate dehydrogenase; PCV, packed cell volume; TLC, total leukocyte count.
Ultrasound of the abdomen showed $9 \times 6 \mathrm{~cm}$ of the right and $4 \times 3 \mathrm{~cm}$ of the left kidneys. Other organs in the abdomen were normal. The echotexture and flows were normal in the right kidney. There was an increase in echotexture with loss of corticomedullary junction of the left kidney, which was suggestive of sequelae of chronic pyelonephritis (-Fig. 2).

Secondary HTN due to renal parenchymal disease was diagnosed, and the patient started on prazosin, metoprolol, and chlorthalidone along with amlodipine. Now the patient is asymptomatic with BP of $130 / 80 \mathrm{~mm} \mathrm{Hg}$.

\section{Discussion}

The early diagnosis and management of preeclampsia or eclampsia in a pregnant woman are very important as it is a high-risk factor for both the mother and fetus. ${ }^{3-5}$ Most frequently the preeclampsia diagnosis is done when the BP is checked during antenatal checkups. However, it depends on how early the BP recording will be done, that is, when the woman comes for confirmation of the pregnancy for the first time, that is, 10 days after the missed period or in the next antenatal visits. Ideally, at every visit, it is better to check the BP.

In this case, first-time HTN was detected at the fifth month of pregnancy, and the patient had to pedal with proteinuria, and at that time the gynecologist was certain about the preeclampsia diagnosis. Fortunately, there were no complications of eclampsia during pregnancy. HTN was also controlled quickly and effectively with nifedipine during pregnancy and cesarean section. Problem with uncontrolled HTN started nearly 15 days after delivery, which was actually due to HTN secondary renal parenchymal disease. In this case, the main discussion is whether the patient had underlined HTN detected during pregnancy or whether she suffered in addition from preeclampsia. It was unlikely that the patient had only preeclampsia as the actual phase of accelerated hypertension

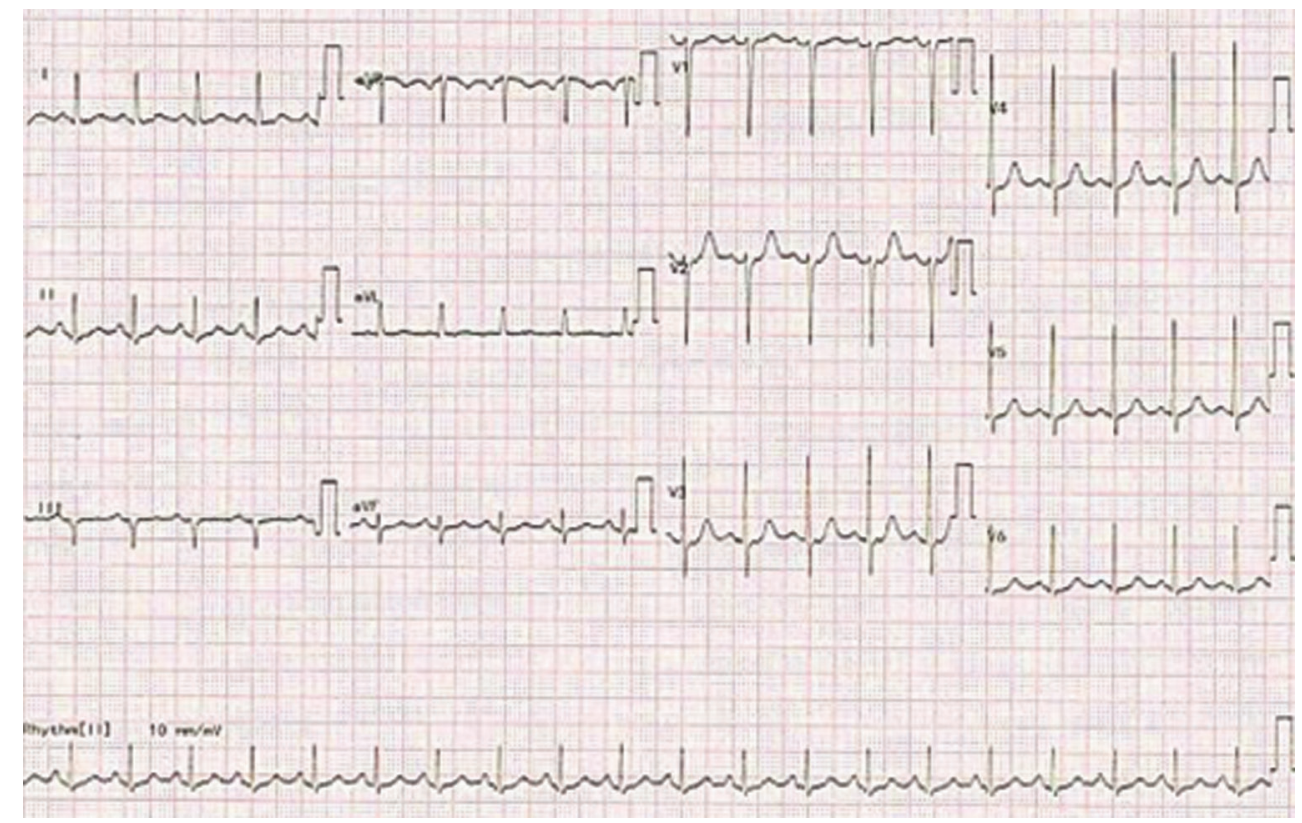

Fig. 1 ECG of the patient. 


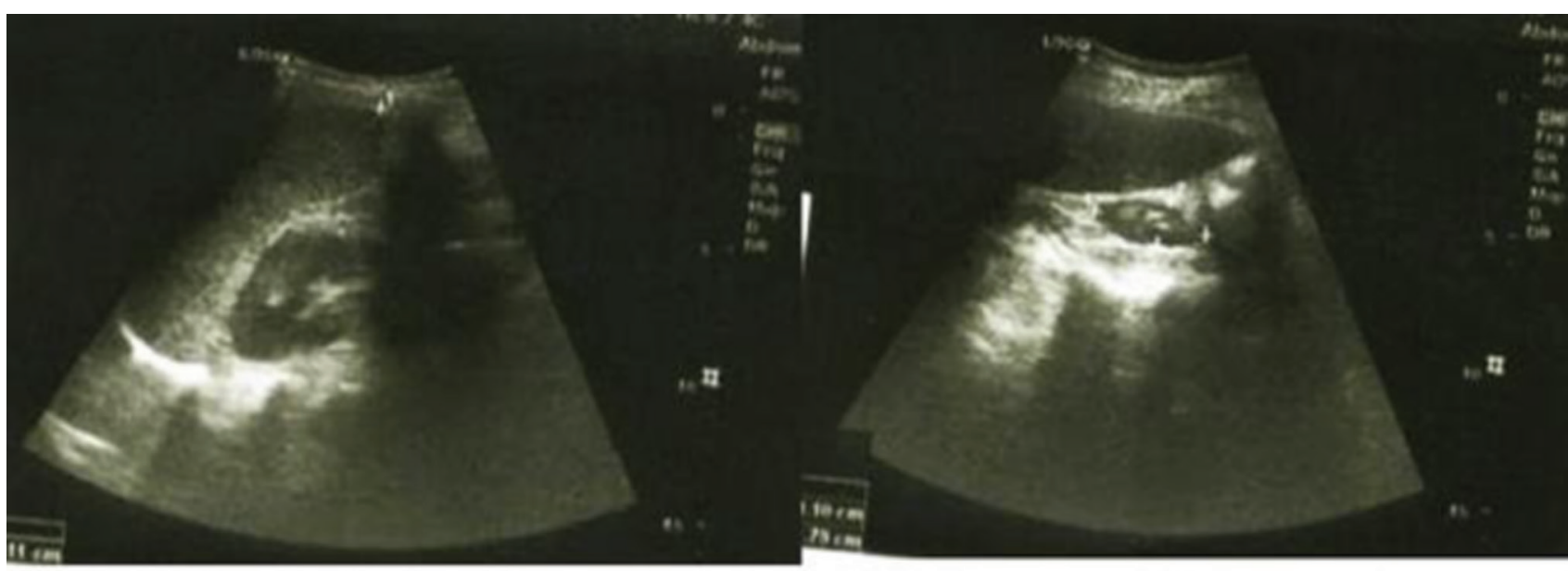

\section{Right Kidney}

\section{Left Kidney}

Fig. 2 Ultrasound images of kidneys.

occurred 2 weeks after delivery, but according to Al-Safi et al, the hypertensive phase main continues in the post-delivery also for few weeks. ${ }^{6}$ The odd point, in this case, is accelerated phase occurred after delivery but not during pregnancy. Even though we think that an expansion of blood volume may have contributed to accelerated phase, but we expect that it occurs around peripartum time rather than 2 weeks later.

If it were only hypertensive heart disease and was detected during pregnancy as BP recording was done only during pregnancy, we expect accelerated HTN is more likely to occur during pregnancy as blood volume expansion is added and in the peripartum period. However, the patient did not have accelerated HTN during pregnancy but only 2 weeks later. Investigations are in favor for chronic renal parenchymal disease leading to secondary hypertension. In this case, further evaluation such as magnetic resonance angiography (MRA) assessment of renal artery stenosis, if the patient permits, is also required. Even though the renal artery stenosis with sunken kidney may not be relevant for management view, but it is better to assess the other kidney for renal artery stenosis.

The patient had significant proteinuria that may be due to hyperperfusion-induced focal segmental disease of the right kidney. Therefore, it is better to wait for a few more weeks and reassess the degree of proteinuria before planning for the biopsy and other causes of proteinuria to evaluate. Actually, a sunken kidney is contraindication to the renal biopsy now.

Another controversy is about when to perform the delivery in these types of patient. Churchill et al have done six articles' review on interventionist versus expectant care for severe preeclampsia between 24 and 34 weeks' gestation and concluded that expected mode of delivery has less morbidity to the fetus. ${ }^{7}$ In our case, cesarean section was done after checking the maturity of the fetus.

Abalos et al done the review on the drug therapy of mild to moderate HTN during pregnancy and found calcium channel blockers and $\beta$-blockers to be useful. ${ }^{8}$ They also found that control of mild to moderate HTN may prevent severe HTN development like in our case in which HTN was under control until the delivery. The gynecologist treated this patient only with nifedipine without $\beta$-blockers (which may cause low birth weight to the baby).

Prenatal exercise is currently recommended to prevent complication of gestational HTN or diabetes. ${ }^{9}$ Shear wave velocity is calculated noninvasively by ultrasound to see the placental elasticity, which is useful in dividing the cases into high or low risk for preeclampsia. ${ }^{10,11}$ Aspirin is recommended for pregnancies with high risk for preeclampsia. ${ }^{12}$ Townsend et al in their reviews suggested that prospective, well-designed studies of predictive markers, preferably in randomized intervention studies and combined through IPD (individual patient data) meta-analyses, are required to develop and validate new prediction models to facilitate the prediction of preeclampsia and minimize further research waste in this field. ${ }^{13}$

\section{Conclusion}

In case of renal artery stenosis, it is always better to record $\mathrm{BP}$ at every visit of antenatal period along with when the patient comes for the planning for pregnancy itself. As in this case whether it is chronic hypertensive disease-complicated pregnancy or really superimposition of preeclampsia can be detected by regular BP checkups. It is advisable to follow preeclamptic patients after delivery until BP normalizes. This is important not only to diagnose other causes of HTN but also to prevent long-term complications of the preeclampsia.

\section{Conflict of Interest}

None.

\section{References}

1 Buschman AR, Rep A. Pre-eclampsia: understanding clinical complexity. Evol Med Public Health 2018;2018(1):211-212

2 Williams, PJ. The genetics of pre-eclampsia and other hypertensive disorders of pregnancy. Best Pract Res Clin Obstet Gynaecol 2011;25(4-4):405-417 
3 Basit S, Wohlfahrt J, Boyd HA. Pre-eclampsia and risk of dementia later in life: nationwide cohort study. BMJ 2018;363:k4109

4 Hollegaard B, Lykke JA, Boomsma JJ. Time from pre-eclampsia diagnosis to delivery affects future health prospects of children. Evol Med Public Health 2017;2017(1):53-66. eCollection 2017

5 Ray JG, Park AL. Pre-eclampsia and the brain. BMJ 2018 Oct 17;363:k4236

6 Al-Safi Z, Imudia AN, Filetti LC, Hobson DT, Bahado-Singh RO, Awonuga AO. Delayed postpartum preeclampsia and eclampsia: demographics, clinical course, and complications. Obstet Gynecol 2011;118(5):1102-1107

7 Churchill D, Duley L, Thornton JG, Moussa M, Ali HS, Walker KF. Interventionist versus expectant care for severe pre-eclampsia between 24 and 34 weeks' gestation. Cochrane Database Syst Rev 2018;10:CD003106

8 Abalos E, Duley L, Steyn DW, Gialdini C. Antihypertensive drug therapy for mild to moderate hypertension during pregnancy. Cochrane Database Syst Rev 2018;10:CD002252
9 Davenport MH, Ruchat SM, Poitras VJ, et al. Prenatal exercise for the prevention of gestational diabetes mellitus and hypertensive disorders of pregnancy: a systematic review and meta-analysis. Br J Sports Med 2018;52(21):1367-1375

10 Fujita Y, Nakanishi TO, Sugitani M, Kato K. placental elasticity as a new non-invasive predictive marker of preeclampsia. Ultrasound Med Biol 20180ct 17. pii: S0301-5629 (18)30405-8.

11 Eastwood KA, Hunter AJ, Patterson CC, Mc Cance DR, Young IS, Holmes VA. Placental vascularization indices and prediction of pre-eclampsia in high-risk women. Placenta 2018;70:53-59

12 Sharma AK, Bhatla N. Aspirin for pregnancies at high risk for preterm pre-eclampsia. Natl Med J India 2018;31(1):26-27

13 Townsend R, Khalil A, Premakumar Y, et al; IPPIC Network. Prediction of pre-eclampsia: review of reviews. Ultrasound Obstet Gynecol 2018 [Epub ahead of print] 